\title{
A Study of Deterministic Positioning of Carbon Nanotubes by Dielectrophoresis
}

\author{
A. ARUN, ${ }^{1,2}$ P. SALET, ${ }^{1}$ and A.M. IONESCU ${ }^{1}$ \\ 1.-Nanolab, Ecole Polytechnique Fédérale Lausanne (EPFL), 1015 Lausanne, Switzerland. \\ 2.-e-mail: anu.arun@epfl.ch
}

A self-limiting dielectrophoresis technique, aimed at deterministically assembling individual or bundles of single-walled carbon nanotubes (SWCNTs) and multiwalled carbon nanotubes (MWCNTs), is experimentally investigated. A limiting resistor is used to control the electric field after the deposition of a single carbon nanotube. The role of some key parameters such as voltage and duration of the deposition with and without the limiting resistor is studied.

Key words: Carbon nanotubes, dielectrophoresis, self-assembly

\section{INTRODUCTION}

Carbon nanotubes (CNTs) are expected to offer outstanding electrical and mechanical properties for solid-state electronic and nano-electro-mechanical (NEM) devices. ${ }^{1} \mathrm{~A}$ major technological challenge is the ability to localize the CNTs on the substrate for controlled device and circuit fabrication. For many applications it is highly desirable to have a dense array of $\mathrm{CNTs}^{2}$; however, in order to systematically study the intrinsic properties of CNTs and build novel sensitive systems ${ }^{3,4}$ it is necessary to control precisely the position of a single CNT. Dielectrophoresis ${ }^{5}$ is defined as the motion of matter caused by polarization effects in a nonuniform electric field. This phenomenon could be used to self-assemble CNTs and has shown tremendous promise in the recent years to align CNTs. ${ }^{6-8}$ Krupke et al. ${ }^{9}$ used this technique, modified with an external circuit that controls the trapping of single carboxyl $(\mathrm{COOH})$ functionalized CNTs on silver electrodes. Banerjee et al. $^{10}$ used a similar technique to trap bundles of SWCNTs. In this paper, we investigate the above technique to trap single or bundles of SWCNTs and MWCNTs. The influence of different parameters such as applied voltage, substrate, electrode material, and an external resistor is studied experimentally. Furthermore, as shown recently by Close et al., ${ }^{11}$ this self-assembly technique

(Received October 1, 2008; accepted March 31, 2009;

published online April 14, 2009) could be adapted to build complementary metal oxide semiconductor (CMOS)-compatible CNT-based devices. It is also advantageous to be able to manipulate both SWCNTs and MWCNTs in order to build devices, as reported by Lee et al. ${ }^{4}$

\section{EXPERIMENT AND RESULTS}

Figure 1 illustrates the process flow and the experimental setup used in this work. Silicon wafers with resistivity of $0.1 \Omega \mathrm{cm}$ to $0.5 \Omega \mathrm{cm}$ with $1-\mu \mathrm{m}$ thick thermal oxide, were used as substrate. $\mathrm{Cr} / \mathrm{Pt}$ $(5 \mathrm{~nm} / 50 \mathrm{~nm})$ electrodes were patterned by standard optical photolithography and lift-off technique. One milligram of SWCNTs produced by chemical vapor deposition (CVD) were dispersed by sonication in $100 \mathrm{~mL}$ deionized (DI) water with sodium dodecyl sulfate (SDS) (1 wt.\%) as a surfactant. The resulting solution was further purified by centrifugation and the clean solution on the top was decanted. A clear transparent solution was obtained by this method. A 5-MHz AC signal, with different peak-to-peak voltages that generate different electric fields, was applied across the electrodes. Around $20 \mu \mathrm{L}$ of the prepared solution was deposited onto the electrodes. The electric field was switched off after $120 \mathrm{~s}$ and the electrodes were cleaned with DI water and dried with $\mathrm{N}_{2}$. All the experiments were performed in the presence of white light. Figure 2 shows the results for different applied electric fields. There was no deposition of tubes when the electric field was very low, in this case $0.33 \mathrm{~V} / \mu \mathrm{m}$; the 
(a)

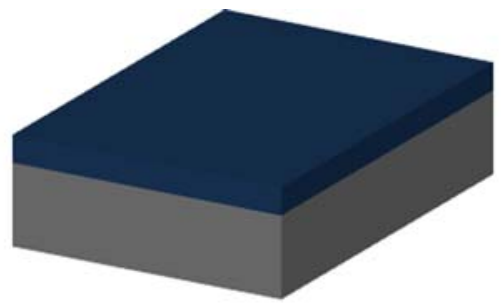

(c)

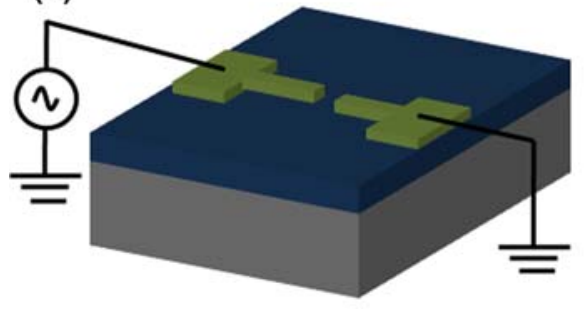

(b)

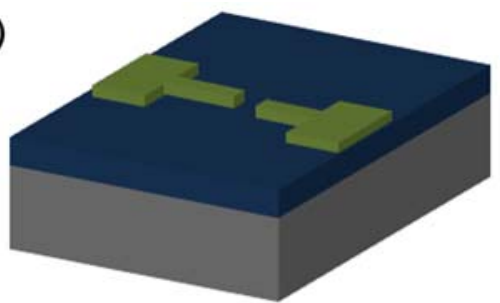

(d)

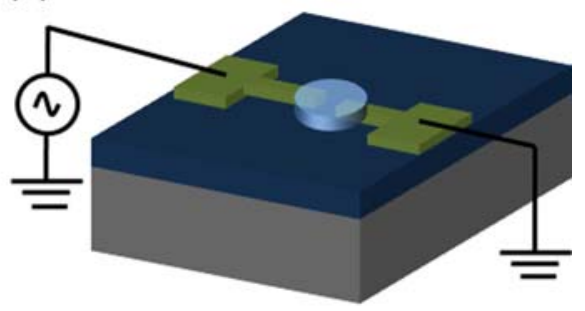

$\mathrm{Cr} / \mathrm{Pt}$

Fig. 1. Process flow of the fabricated structure and the experimental setup used: (a) $\mathrm{Si} / \mathrm{SiO}_{2}(1 \mu \mathrm{m})$ substrate; $(\mathrm{b}) \mathrm{Cr} / \mathrm{Pt}(5 \mathrm{~nm} / 50 \mathrm{~nm})$ electrodes patterned by optical lithography and lift-off; (c) AC electric field applied across the electrodes; (d) $20 \mu \mathrm{L}$ CNT solution is deposited across the electrodes for a duration of $120 \mathrm{~s}$, then the electric field is switched off and the wafer is washed with DI water and dried in $\mathrm{N}_{2}$.
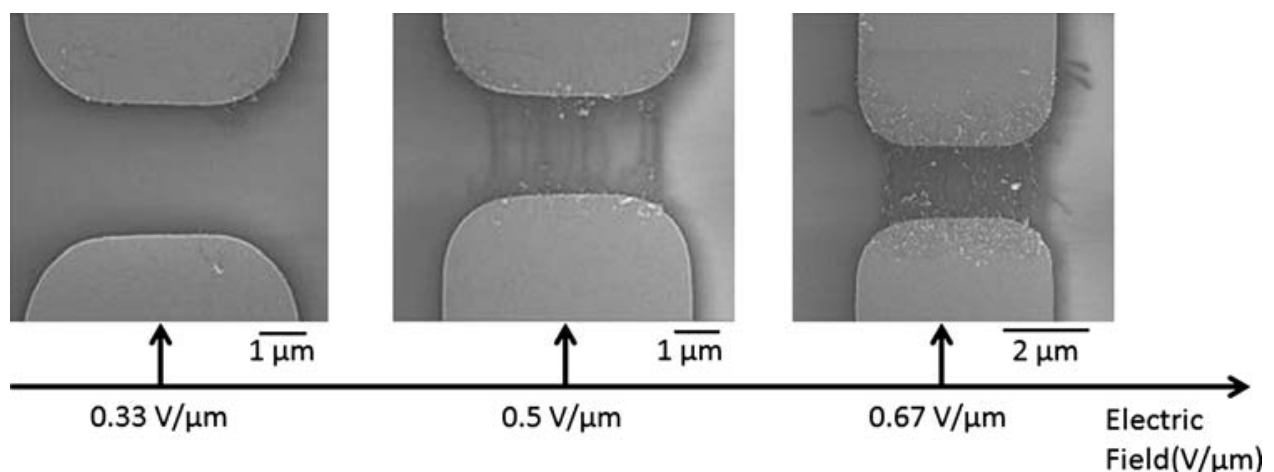

Fig. 2. Effect of electric field: scanning electron microscope (SEM) images of the structures on which dielectrophoresis was performed with the setup shown in Fig. 1c with different voltages applied across the electrode. There was no deposition of tubes when the electric field was very low, in this case $0.33 \mathrm{~V} / \mu \mathrm{m}$; the minimum electric field for the deposition of CNTs was found to be $0.5 \mathrm{~V} / \mu \mathrm{m}$. With increased electric field $(0.67 \mathrm{~V} / \mu \mathrm{m})$, the number of trapped tubes increased.

minimum electric field to start deposition of CNTs was found to be around $0.5 \mathrm{~V} / \mu \mathrm{m}$. With increased electric field $(0.67 \mathrm{~V} / \mu \mathrm{m})$, the number of tubes trapped increased. These values compare well with the ones reported by Chung et al. ${ }^{12}$ The trapped SWCNTs included both metallic and semiconducting tubes. The average diameter of these tubes was found to be $14 \mathrm{~nm}$ by performing tapping-mode atomic force microscope (AFM) measurements. Presence of even a single metallic CNT within the bundle causes the whole bundle to behave as metallic CNTs by dominating the dielectric constant, ${ }^{13}$ enabling positive dielectrophoresis.

With the above-mentioned technique it is also possible to trap a single bundle of SWCNTs by reducing the $\mathrm{CNT}$ concentration or deposition time, and/or by reducing the electrode width via e-beam lithography. However, reducing the CNT concentration reduces the yield of the process. ${ }^{8}$ An alternative technique would be to have the ability to stop further deposition of CNTs automatically once a single CNT has been trapped. This can be achieved by introducing an external resistor in series into the setup used in Fig. 1c, as shown in Fig. 3a. The effectiveness of the limiting resistor is demonstrated in Fig. 3b-e. For the same deposition conditions, without the self-limiting resistor, arrays of CNTs were aligned across the two electrodes. In contrast, only with the self-limiting resistor has it been possible to trap a single tube.

In order to understand the role of the external resistor let us consider the configuration shown in Fig. 3a, as a resistor divider circuit. Here we have assumed that the impedance of the circuit at $5 \mathrm{MHz}$ 


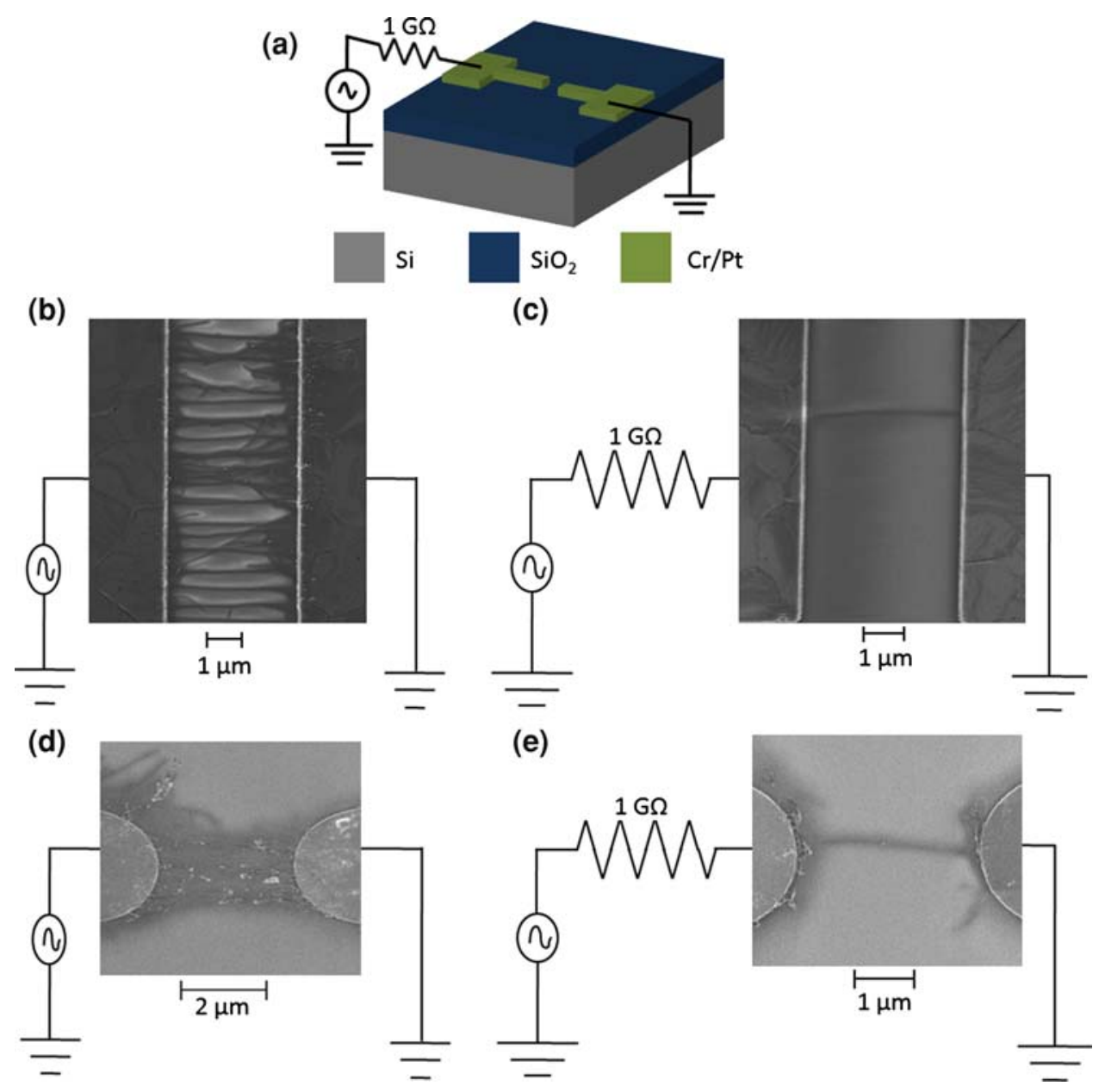

Fig. 3. (a) Modified setup to perform dielectrophoresis, using a self-limiting resistor. (b, c) SEM images of the structures on which dielectrophoresis is performed, with and without a self-limiting resistor, respectively. Both the structures have the same electrode shape and spacing. An array of CNTs is trapped without a self-limiting resistor. An individual bundle of SWCNT is trapped with a self-limiting resistor. (d, e) SEM images of the structures on which dielectrophoresis is performed, with and without a self-limiting resistor, respectively, with different electrode shape and dimensions compared with the structure in (b) and (c). Both structures have the same electrode shape and spacing.

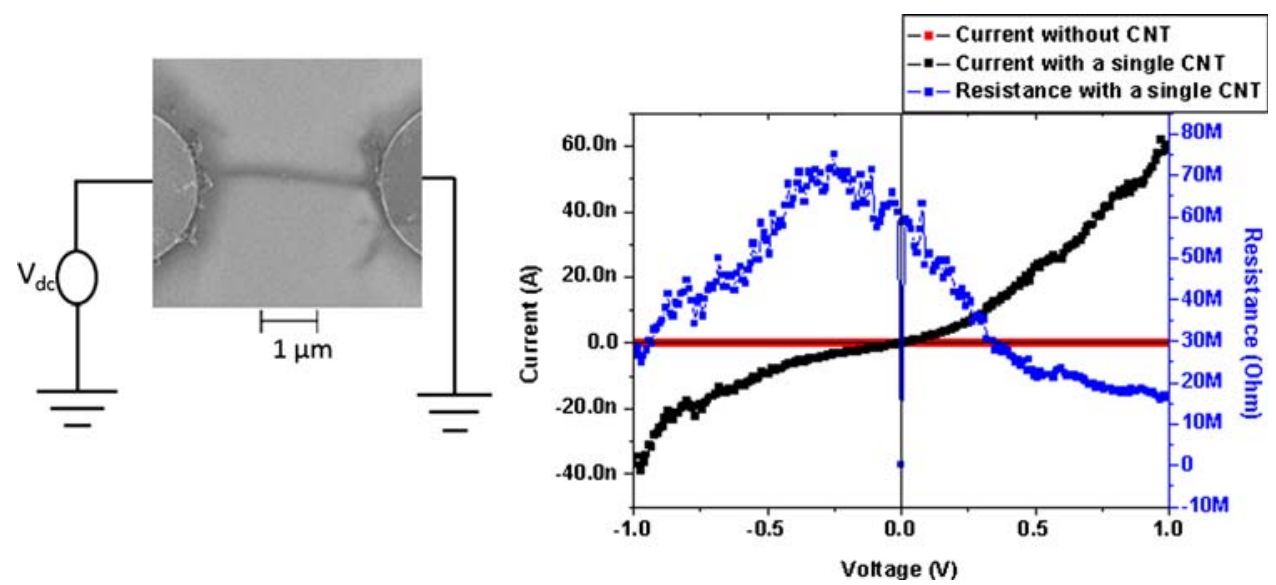

Fig. 4. Two-probe DC measurements performed on individual CNTs. A DC current corresponding to resistance values in the range of $30 \mathrm{M} \Omega$ to $80 \mathrm{M} \Omega$ is measured when such an experiment is performed on ten assembled devices.

is approximately the same as that at DC. ${ }^{14}$ After deposition of a single CNT, the electric field across the electrodes where the CNTs are to be deposited decreases. This was confirmed by $I-V$ measurements performed before and after the deposition of the CNT (Fig. 4). After the deposition of a single 

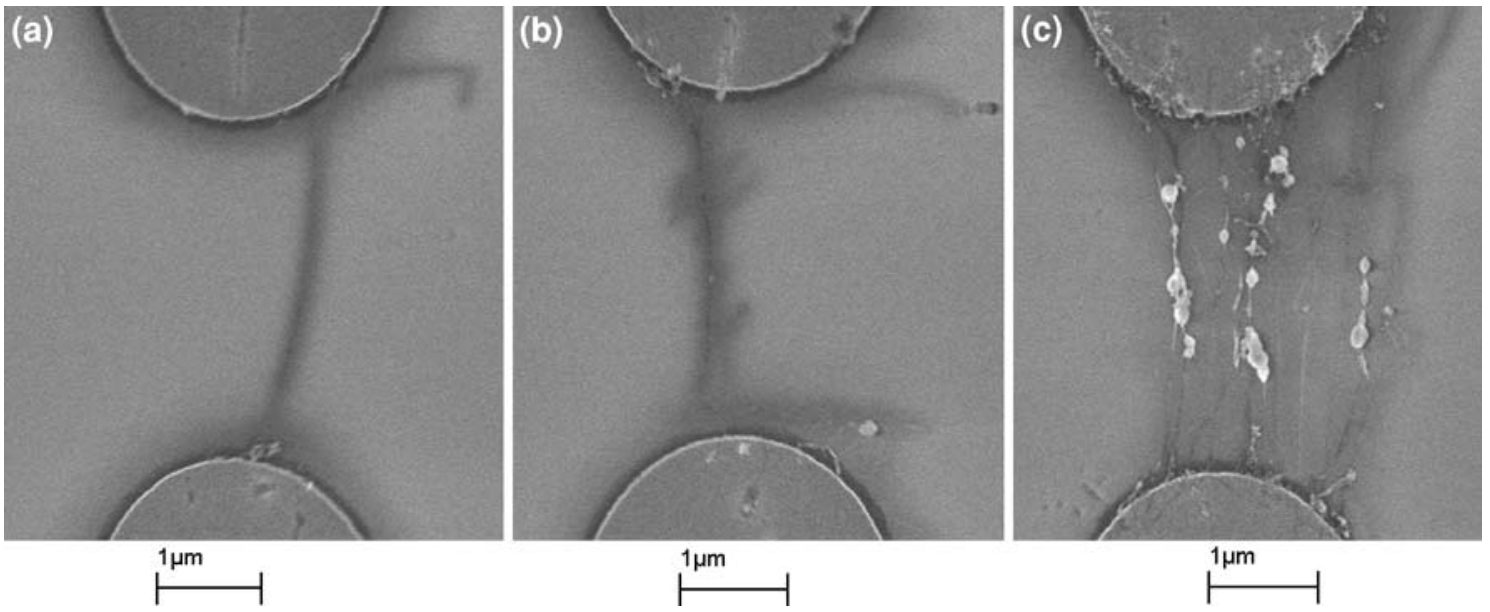

Fig. 5. Effect of deposition time when using the configuration in Fig. 3a for the experiment. SEM images obtained with the same deposition conditions, varying the duration of deposition: (a) $120 \mathrm{~s}$, (b) $300 \mathrm{~s}$, and (c) $600 \mathrm{~s}$. The external resistor limits the deposition of many tubes after deposition of a single tube but, even with a $1 \mathrm{G} \Omega$ external resistor, this limiting mechanisms is not ideal since the contact resistance of the tubes is relatively high.

CNT, we measure resistances in the range of $30 \mathrm{M} \Omega$ to $80 \mathrm{M} \Omega$, with measurements done on ten devices. This implies that there was an effective electrical contact between the CNT and the electrode after deposition of a CNT. Since the external resistor used (1 G $\Omega$ ) was larger than this value, after deposition of a single CNT, the voltage drop across the external resistor starts to dominate. In other words, the electric field across the electrodes where CNTs are to be deposited was strongly reduced, which prevented further deposition of CNTs. We have also confirmed this by carrying out deposition for different durations. If the external resistor would completely cut off the electric field after the deposition of a single CNT, the CNT deposition should be independent of the duration of deposition (we should always have a single CNT aligned across the electrodes). Figure 5 shows the results obtained for different deposition times. We observed that, with increasing deposition time, the number of trapped CNTs increases slightly but seems to saturate after a few tubes are trapped (probably because more tubes in parallel further reduce the local field for dielectrophoresis). This can be explained by the fact that the electric field across the electrodes is reduced after deposition of the CNTs, but not completely cut off. Hence there is a lower yet finite probability to trap more tubes for long deposition times. With this technique we can relax the deposition parameters such as deposition time and concentration; even with relatively wide electrodes (several $\mu \mathrm{m}$ ) trapping of a single tube can be controlled.

The same technique and conditions have been used to deposit single MWCNT. MWCNTs produced by CVD and the arc-discharge technique were used. The experiments performed with MWCNTs were similar to those of the SWCNTs except that, during solution preparation for the
MWCNTs, no surfactant was used. Two milligrams of CVD tubes were dispersed in $100 \mathrm{~mL}$ DI water, whereas $5 \mathrm{mg}$ arc-discharge tubes were dispersed in $100 \mathrm{~mL}$ DI water. The resulting solution was sonicated and centrifuged, and the resulting clean solution on the top was decanted to obtain a clean well-dispersed solution. Since the fabrication technique for each of the tubes used in our experiments (CVD SWCNT, CVD MWCNT, and arc-discharge MWCNT) is different, the resulting purity and density of the tubes are different. Hence the concentration of the solution was optimized for each of them. Figure $6 a, c$ and $b$, d shows the results obtained with MWCNTs produced by CVD, with and without the self-limiting resistor, respectively. For the case without the self-limiting resistor a large number of tubes were deposited, whereas with the self-limiting resistor only a single tube was trapped, thus demonstrating the effectiveness of the technique. Due to their curled-up nature, the CVD tubes were not well dispersed in the solution and lumps were seen in some cases. This reduces the probability of having a single tube that is well aligned. This problem could probably be solved with advances in the technology used in the growth of CVD tubes to produce high-quality tubes, or alternatively by using arc-discharge tubes (Fig. 7). With arc-discharge tubes we were able to obtain relatively straight tubes, although this came at the price of increased carbon soot, thus making it difficult to prepare a clean dispersed solution. The increased carbon soot is a result of the inherent production technique. Also the length variation of the CNTs (produced by the arc-discharge technique) used in this work was large; we have carried out experiments with CNTs having lengths in the range of $100 \mathrm{~nm}$ up to $1 \mu \mathrm{m}$, which further reduces the chance of trapping a single $\mathrm{CNT}$, hence resulting in poor yield for these CNTs. 

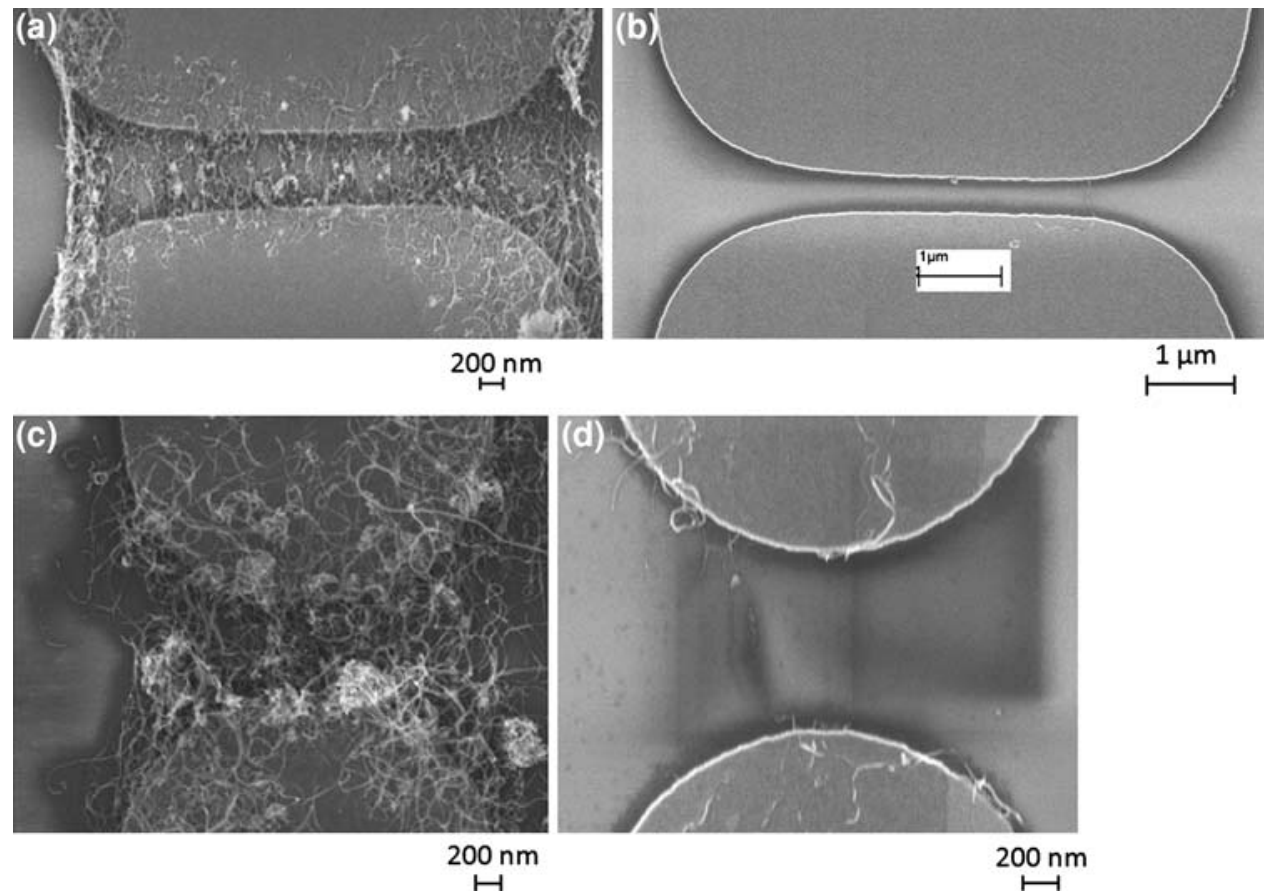

Fig. 6. Dielectrophoresis performed without and with a self-limiting resistor on CVD MWCNTs. (a, b) Dielectrophoresis performed on two sets of electrodes without and with a self-limiting resistor, respectively. (c, d) Dielectrophoresis performed on another set of electrodes with a different electrode shape compared to (a), without and with a self-limiting resistor, respectively. SEM images show that, without a self-limiting resistor, a large number of tubes are aligned across the electrodes, whereas with a self-limiting resistor, a single tube is aligned.
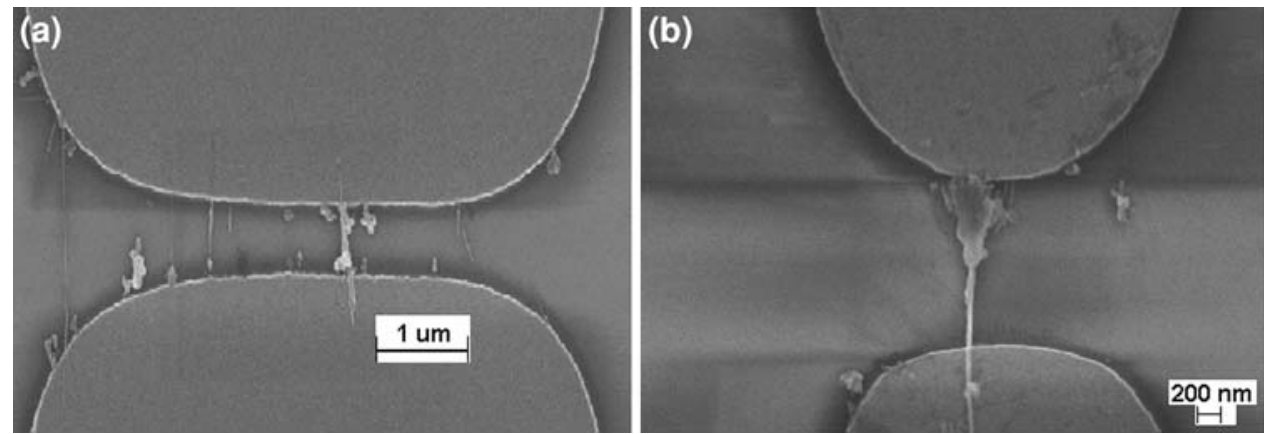

Fig. 7. Dielectrophoresis performed with and without a self-limiting resistor using MWCNTs produced by an arc-discharge technique. SEM images show that, without a self-limiting resistor, a large number of tubes are aligned across the electrodes whereas, with a self-limiting resistor, a single tube is aligned. (a) No self-limiting resistor used, (b) self-limiting resistor used. Also seen in these images are some dirt-like particles, which come from the inherent production technique for the arc-discharge tubes.

\section{INFLUENCE OF ELECTRODE MATERIAL}

For the self-limiting technique to work well, it is desirable to have a low-resistive contact between the CNT and the electrodes. In all the processes mentioned above we used $\mathrm{Cr} / \mathrm{Pt}(5 \mathrm{~nm} / 50 \mathrm{~nm})$ as the electrode material. We carried out the same experiment using Pd electrodes in place of Pt electrodes. $\mathrm{Pd}$ has a workfunction close to that of the CNT, which could further reduce the contact resistance. We did not see any significant change in the contact behavior with $\mathrm{Pd}$ as electrode material. The high contact resistance and apparent insensitivity to the electrode material can be attributed to the fact that our tubes are directly deposited on top of the electrodes and measurements are reported without any further contact processing, which results in nonlinear contacts (Fig. 4). It follows that the contact resistance between the tube and the electrodes by dielectrophoresis is good and efficient enough to self-limit the deposition process, but needs supplementary processing and local optimization after the dielectrophoretic step in order to build a functional electronic device.

\section{INFLUENCE OF SUBSTRATE}

We investigate the influence of substrates on dielectrophoresis by performing identical experiments on four different substrates: (i) low-resistivity 


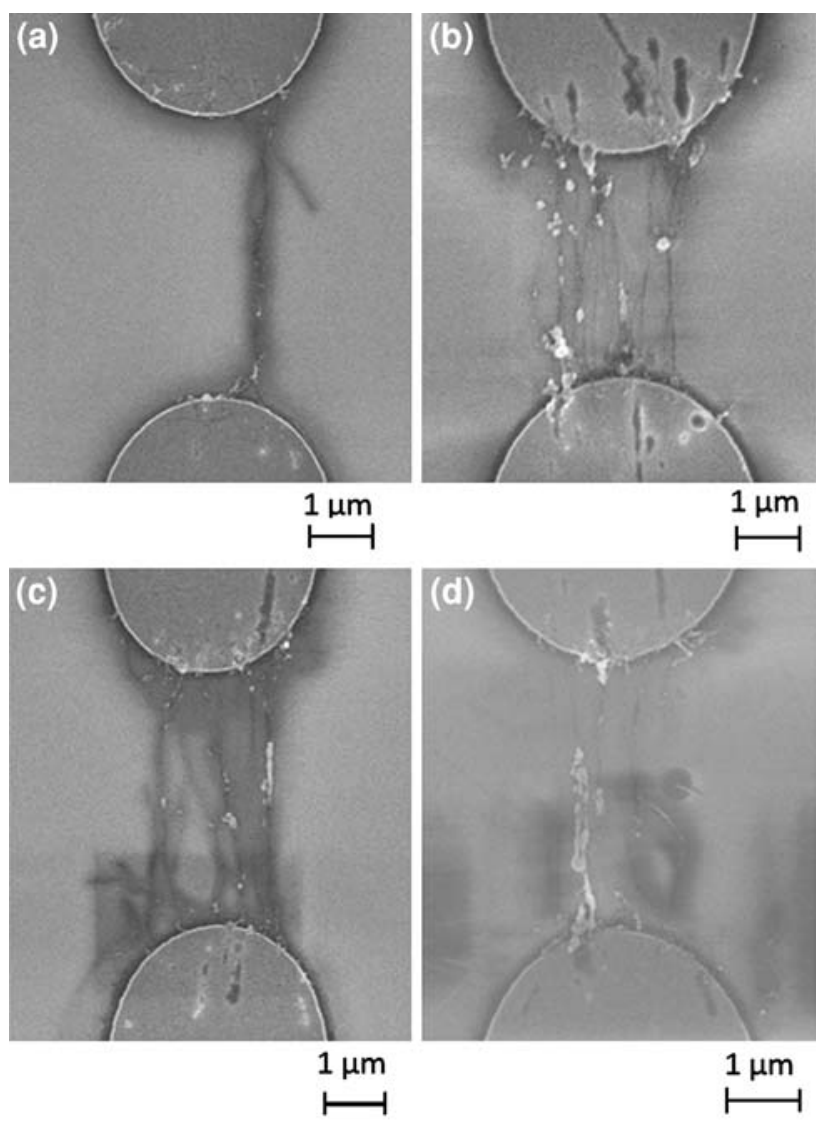

Fig. 8. Study on the influence of substrate. Dielectrophoresis experiments performed with the self-limiting resistor on different substrates: (a) low-resistivity $\mathrm{Si}(0.1 \Omega \mathrm{cm}$ to $0.5 \Omega \mathrm{cm}) / \mathrm{SiO}_{2}(1 \mu \mathrm{m})$ substrate, (b) low-resistivity $\mathrm{Si}(0.1 \Omega \mathrm{cm}$ to $0.5 \Omega \mathrm{cm}) / \mathrm{SiO}_{2}(10 \mu \mathrm{m})$ substrate, (c) high-resistivity $\mathrm{Si}(10 \mathrm{k} \Omega \mathrm{cm}) / \mathrm{SiO}_{2}(1 \mu \mathrm{m})$ substrate, and (d) quartz substrate. Influence of substrate on the required electric field for deposition of CNT: for the same applied voltage electric field across the electrodes it is higher for highly insulating substrates (b, c and d) compared with the case of a low-resistivity silicon substrate (a), hence more CNTs are trapped in these cases.

silicon substrates with resistivity of $0.1 \Omega \mathrm{cm}$ to $0.5 \Omega \mathrm{cm}$ with $1-\mu \mathrm{m}$ wet oxide (sub1), (ii) low-resistivity silicon substrates with resistivity of $0.1 \Omega \mathrm{cm}$ to $0.5 \Omega \mathrm{cm}$ with $10-\mu \mathrm{m}$ wet oxide (sub2), (iii) highresistivity silicon substrates with resistivity of $10 \mathrm{k} \Omega \mathrm{cm}$ with $1-\mu \mathrm{m}$ wet oxide (sub3), and (iv) quartz substrates (sub4). Substrates sub2, sub3, and sub4 can be categorized as insulating compared with sub1. Figure 8 summarizes the results. Due to lower substrate losses in the cases of sub2, sub3, and sub4, for the same voltage applied across the electrodes, the electric field is higher for these substrates (Fig. 8b, c and d) compared with the case of sub1 (Fig. 8a). Hence more CNTs are trapped in the case of sub2, sub3, and sub4. By optimizing the applied voltage and using the self-limiting technique we were able to trap individual tubes on any kind of substrate. Empirically, the optimum voltage (the applied voltage that generates adequate electric field to trap CNTs but is not large enough to cause hydrolysis of the solvent or trap a large number of tubes) for insulating substrates (sub2, sub3, and sub4) was found to be $V_{\text {high_res }} \cong$ $V_{\text {low_res }} / 1.5$. These values need further extensive investigations.

\section{INFLUENCE OF SURFACTANT FOR SWCNT}

The surfactant wraps the CNTs and disperses them well in the solvent, but it does alter the properties of the CNTs, ${ }^{15}$ which is not desirable. Hence it would be ideal not to use surfactant to disperse the CNTs in the solvent. It is relatively easy to disperse MWCNTs in any solvent, but this is not the case for SWCNTs. We did some experiments to disperse SWCNTs in DI water without any surfactant. We used the Bandelin Sonopuls ultrasonic sonicator from Amplichron ${ }^{\circledR}$ system at a continuous output power of $100 \%$ for $30 \mathrm{~min}$ to disperse SWCNTs without any surfactant in DI water, whereas when we used the surfactant, the SWCNTs were dispersed in the same sonicator at $30 \%$ power for only $5 \mathrm{~min}$. The solutions were cooled to room temperature before proceeding with dielectrophoresis experiments. Figure 9 compares the results obtained in the two cases. Even with relatively large sonication power the SWCNTs were not well dispersed when no surfactant was used, hence resulting in varied morphology as opposed to the case when surfactant was used. This is a fundamental limitation to the deposition of pristine SWCNTs using the dielectrophoresis technique.

\section{YIELD}

Table I shows some preliminary results on the yield obtained from our experiments. As seen from the table it is relatively easy to trap an array of CNTs across two electrodes, but the yield is lower for trapping a single tube. The higher yield for the SWCNTs compared with the MWCNTs is first attributed to the relatively uniform length distribution of the SWCNTs used in our experiments compared with that of the MWCNTs, rather than to the influence of tube resistance. Note that the SWCNTs used in our experiments were of high quality in terms of purity compared with the MWCNTs, which results in a higher density of tubes per unit of volume, hence improving the yield of self-assembly. Another possible influence comes from the nature of contact formed between the tubes and the electrodes. In fact, in the case of SWCNTs we trapped bundles of tubes, resulting in low-resistive path across the electrodes compared with the case of MWCNTs, where we have only one tube and higher contact resistance. These data are based on experimental results from 20 trials under optimal deposition conditions. It is clear that further extensive investigations are needed to decouple the impact of various effects on the CNT self-assembly yield. 

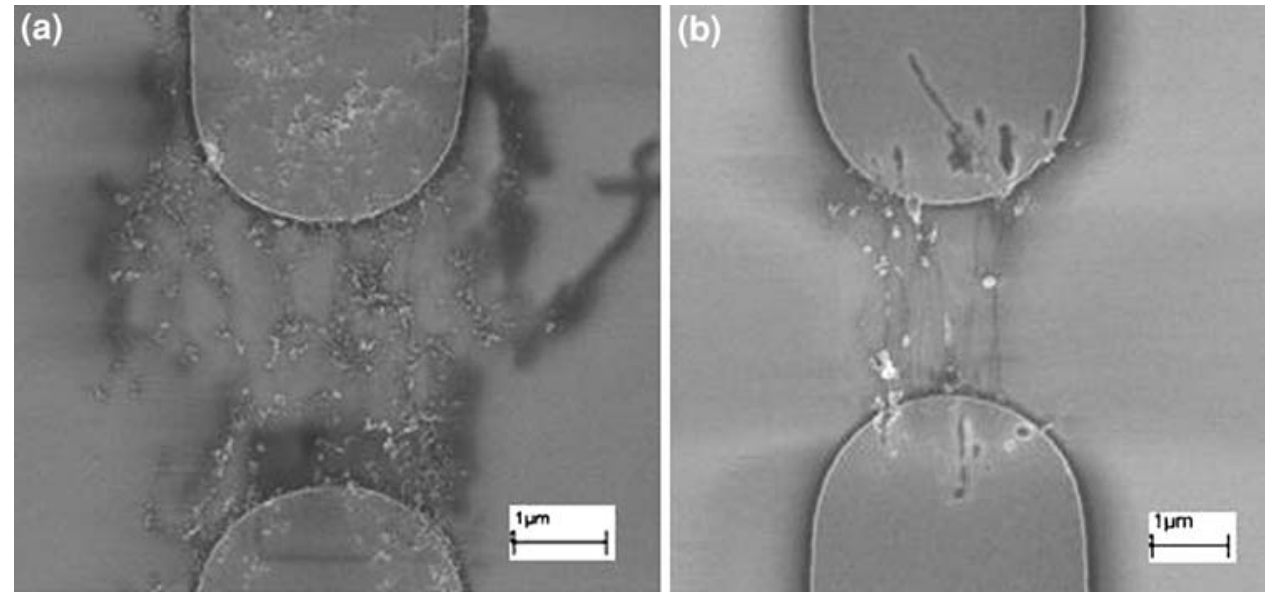

Fig. 9. Effect of surfactant on the dispersion of the SWCNTs. (a) Dielectrophoresis performed using SWCNTs dispersed in DI water without any surfactant. (b) Dielectrophoresis performed using SWCNTs dispersed in DI water with SDS as surfactant. The SWCNTs are not very well dispersed when surfactant is not used, hence resulting in varied morphology as compared with the case when surfactant is used.

Table I. Yield Obtained with Different CNTs for Dielectrophoresis With and Without a Self-Limiting Resistor

\begin{tabular}{lccc}
\hline & $\begin{array}{c}\text { Probability That } \\
\text { There Is Only One CNT } \\
\text { Carbon Nanotube }\end{array}$ & $\begin{array}{c}\text { There Are CNTs Aligned as an Array } \\
\text { (With Self-Limiting Resistor) (\%) }\end{array}$ & $\begin{array}{c}\text { Probability That } \\
\text { (Without Self-Limiting Resistor) (\%) }\end{array}$ \\
\cline { 3 - 4 } SWCNT (CVD) & $\sim 70$ & $>90$ \\
MWCNT (CVD) & $\sim 30$ & $>90$ \\
MWCNT (Arc Discharge) & $\sim 10$ & $>75$ \\
\hline
\end{tabular}

\section{CONCLUSIONS}

An experimental study based on the self-limiting dielectrophoresis technique has been reported. It was found that it provides significantly improved control to trap single or bundles of SWCNTs and MWCNTs compared with the traditional dielectrophoresis method without a limiting resistor. The experimental parameters that influence the CNT trapping are: (i) the electric field between the metal electrodes and the ratio between the self-limiting resistance and the resistance of the tubes (including contact series resistances), (ii) the duration of deposition, (iii) the substrate resistivity, and (iv) the surfactant. For all our experiments, the reported yield was better for SWCNTs compared with MWCNTs. The investigated technique has the merit of enabling trapping of CNTs at desired locations, with control of the number of assembled tubes, which paves the way for future design and fabrication of CNT-based devices and circuits.

\section{ACKNOWLEDGEMENTS}

This project is funded by NANO-RF, FP6 European project. We would like to thank Dr. Arnaud Margez and Prof. Forro from EPFL for providing all the CNTs used in our experiments.

\section{REFERENCES}

1. R. Saito, G. Dresselhaus, and M.S. Dresselhaus, Physical Properties of Carbon Nanotubes (London: Imperial College Press, 1998).

2. S.J. Kang, C. Kocabas, T. Ozel, M. Shim, N. Pimparkar, M.A. Alam, S.V. Rotkin, and J.A. Rogers, Nat. Nanotechnol. 2, 230 (2007). doi:10.1038/nnano.2007.77.

3. V. Sazonova, Y. Yaish, H. Ustunel, D. Roundy, T.A. Arias, and P.L. McEuen, Nature 431, 284 (2004). doi:10.1038/ nature02905.

4. D.S. Lee, S.J. Park, S.D. Park, Y.W. Park, M. Kemell, M. Ritala, J. Svensson, M. Jonson, and E.B. Campbell, Appl. Phys. Lett. 89, 233107 (2006). doi:10.1063/1.2402 119.

5. H.A. Pohl, J. Appl. Phys. 29, 1182 (1958). doi:10.1063/1.17 23398.

6. X.Q. Chena, T. Saito, H. Yamada, and K. Matsushige, Appl. Phys. Lett. 78, 3714 (2001). doi:10.1063/1.1377627.

7. A. Vijayaraghavan, S. Blatt, D. Weissenberger, M. Oron-Carl, F. Hennrich, D. Gerthsen, H. Hahn, and R. Krupke, Nano Lett. 7, 1556 (2007). doi:10.1021/nl0703727.

8. T. Schwamb, T.Y. Choi, N. Schirmer, N.R. Bieri, B. Burg, J. Tharian, U. Sennhauser, and D. Poulikakos, Nano Lett. 7, 3633 (2007). doi:10.1021/nl071853t.

9. R. Krupke, F. Hennrich, H.B. Weber, B. Beckmann, O. Hamper, S. Malik, M.M. Kappes, and H.V. Lohneysen, Appl. Phys. Adv. Mater. 76, 397 (2003).

10. S. Banerjee, B.E. White, L. Huang, B.J. Rego, S. O'Brien, and I.P. Herma, J. Vac. Sci. Technol. B 24, 3173 (2006). doi:10.1116/1.2387155.

11. G.F. Close, S. Yasuda, B. Paul, S. Fujita, and H.S.P. Wong, Nano Lett. 8, 706 (2008). doi:10.1021/nl0730965. 
12. J. Chung, K.H. Lee, J. Lee, and R.S. Ruoff, Langmuir 20, 3011 (2004). doi:10.1021/la035726y.

13. R. Krupke, F. Hennrich, H.V. Lohneysen, and M. Kappes, Science 301, 344 (2003). doi:10.1126/science.1086534.
14. A. Tselev, M. Woodson, C. Qian, and J. Liu, Nano Lett. 8, 152 (2007). doi:10.1021/nl072315j.

15. Z.B. Zhanga, X.J. Liu, E.B. Campbell, and S.L. Zhang, J. Appl. Phys. 98, 056103 (2005). doi:10.1063/1.2037866. 